\title{
Interferometric Observations of Complex Organic Molecules
}

\author{
Sheng-Yuan Liu \\ Academia Sinica, Institute of Astronomy and Astrophysics, \\ Taipei, Taiwan \\ email: syliu@asiaa.sinica.edu.tw
}

\begin{abstract}
Rich molecular complexity has been shown to exist in star forming regions primarily through millimeter to submillimeter single-dish spectral line surveys or searches over the years. With a few case studies, we demonstrate that interferometric observations are a powerful tool for advancing our knowledge of the chemical processes related to the formation of complex organic molecules in these regions. In particular, interferometers are sensitive to compact structures, such as those of the so called "hot molecular core" sources where complex organics are almost exclusively found. The high angular resolutions achieved by interferometers allow us to better spatially discriminate chemical differentiation and, possibly, evolutionary effects.
\end{abstract}

Keywords. astrochemistry - ISM: abundances - ISM: individual (Orion KL, Sgr B2, G9.62 + 0.13) — line: identification

\section{Introduction}

To date more than 130 molecular species have been detected in the interstellar and circumstellar environment. Observations have shown that while simpler and long chain carbon molecules are often found to be abundant in diffuse/dark clouds, complex and more saturated organic species, such as vinyl cyanide $\left(\mathrm{C}_{2} \mathrm{H}_{3} \mathrm{CN}\right)$, ethyl cyanide $\left(\mathrm{C}_{2} \mathrm{H}_{5} \mathrm{CN}\right)$, dimethyl ether $\left(\left(\mathrm{CH}_{3}\right)_{2} \mathrm{O}\right)$, methyl formate $\left(\mathrm{HCOOCH}_{3}\right)$, etc. are found predominantly or even exclusively in the so-called "hot molecular cores," or HMCs. HMCs are dense $\left(n_{\mathrm{H}_{2}}>10^{6} \mathrm{~cm}^{-3}\right)$ and warm $(\mathrm{T}>100 \mathrm{~K})$ molecular condensations often associated with molecular masers, $\mathrm{H}$ II regions, or embedded near-infrared sources. These signatures suggest HMCs are active massive star-forming regions, possibly at the earliest phase of star formation (Kurtz et al. 2000 and this volume).

The formation mechanisms of complex organic species are of great interest. This is particularly true for those molecules of potential biological importance. The understanding of such molecular synthesis may give profound insight into the pervasiveness of organic chemistry in the Universe or even how life started on Earth. Based on models with extensive reaction networks, quiescent gas-phase reactions have quite successfully accounted for the observed molecular composition of diffuse/dark clouds. On the other hand, it appears very unlikely that such processes can explain the formation of complex organic species in the large abundances found toward HMCs. Surface processing and gas-grain interactions were often restored to explain the enhanced abundances of complex organics (e.g., Miao et al. 1995; Mehringer \& Snyder 1996). Detailed quantitative models for grain-surface molecular formation, however, remain difficult to develop due to the lack of our understanding of grain surfaces, molecular mobilities, desorption processes, etc.

To better understand surface reaction processes and their relative importance with respect to gas-phase reactions for molecular formation, two types of comparison may help in establishing guidelines (Turner \& Apponi 2001; see also Hollis, this volume). The first 
comparison is to utilize backbone chemistry: comparison among species with a common backbone or sharing similar structural elements but different degrees of saturation may provide clues to the efficiency of hydrogenation on grain surfaces. Comparison of families with different backbones, on the other hand, may shed light on the mobility of heavy atoms or small functional groups on grain surfaces. Second, complex molecules can form isomeric families, in which the same number and type of atoms can form more than one molecular species. Relative abundances within an isomeric family may contain information on the rearrangement processes on grains and on the relative desorption efficiencies of the various members.

Past molecular line surveys, mainly carried out by single-dish telescopes toward limited lines of sight, have established the paradigm for investigating relative abundances of various molecular species in star-forming regions, including those sharing common backbones or that are members of the same isomeric family (e.g., Blake et al. 1987; Turner 1991; Schilke et al. 1997). Nevertheless, the angular resolutions achieved by these studies are often sufficiently coarse that different species, though they trace different cloud components, are found within the same beam and thus cannot be easily distinguished spatially or even kinematically. The derived relative abundances between various species are therefore averages over different cloud components.

To remedy the above difficulties, observations at higher angular resolution, which can be naturally achieved by interferometers, are highly desired. This is especially important for studying HMCs, given that these sources are often located at substantial distances, typically several kiloparsecs away. In addition to the their critical imaging capabilities, interferometers also act as spatial filters to remove extended structures, hence better matching and isolating the emission from sources that are compact in nature, like HMCs.

We highlight here two case studies of complex organics carried with interferometric observations, mainly toward HMCs. The first is the imaging of $\mathrm{HCOOH}$ and $\mathrm{CH}_{3} \mathrm{COOH}$; the second involves the aperture synthesis imaging of the $\mathrm{C}_{2} \mathrm{H}_{4} \mathrm{O}$ isomeric triplet. We also present a new observational example of chemical differentiation between complex nitrogen- and oxygen-bearing species in star-forming cores.

\section{The Imaging of $\mathrm{HCOOH}$ and $\mathrm{CH}_{3} \mathrm{COOH}$}

Among the complex organic species, formic acid $(\mathrm{HCOOH})$ and the related species acetic acid $\left(\mathrm{CH}_{3} \mathrm{COOH}\right)$ are two of great interest. $\mathrm{HCOOH}$ is the simplest organic acid; while acetic acid and $\mathrm{HCOOH}$ share common structural elements with glycine $\left(\mathrm{NH}_{2} \mathrm{CH}_{2} \mathrm{COOH}\right)$, the simplest and biologically important amino acid. In the laboratory a bimolecular synthesis of $\mathrm{NH}_{2} \mathrm{CH}_{2} \mathrm{COOH}$ can occur when acetic acid combines with $\mathrm{NH}_{2}^{+}$. $\mathrm{CH}_{3} \mathrm{COOH}$ is also important for astrochemical studies because it contains the elusive $\mathrm{C}-\mathrm{C}-\mathrm{O}$ backbone; interstellar molecules with this structure appear to have less favorable formation routes than their counterparts with the $\mathrm{C}-\mathrm{O}-\mathrm{C}$ backbone (Millar et al. 1988). For example, an isomer of acetic acid, methyl formate $\left(\mathrm{HCOOCH}_{3}\right)$, has the $\mathrm{C}-\mathrm{O}-\mathrm{C}$ backbone and is detected ubiquitously in a host of HMCs.

Interstellar $\mathrm{HCOOH}$ was first reported by Zuckerman, Ball, \& Gottlieb (1971) and by Winnewisser \& Churchwell (1975), both toward Sgr B2; and has been subsequently identified in single-dish telescope spectral line surveys since then (e.g., Turner 1991; Ziurys, \& McGonagle 1993), primarily toward two massive star forming regions-Sgr B2 and Orion KL. More recently, $\mathrm{HCOOH}$ was observed in comet Hale-Bopp (Crovisier \& Bockelée-Morvan 1999) and tentatively identified in interstellar ice absorption spectra (Schuttee et al. 1996, 1999). $\mathrm{CH}_{3} \mathrm{COOH}$, on the other hand, was not found in ISM until the first detection with an interferometric array (Mehringer et al. 1997). 
G31.41+0.31

G10.47+0.03
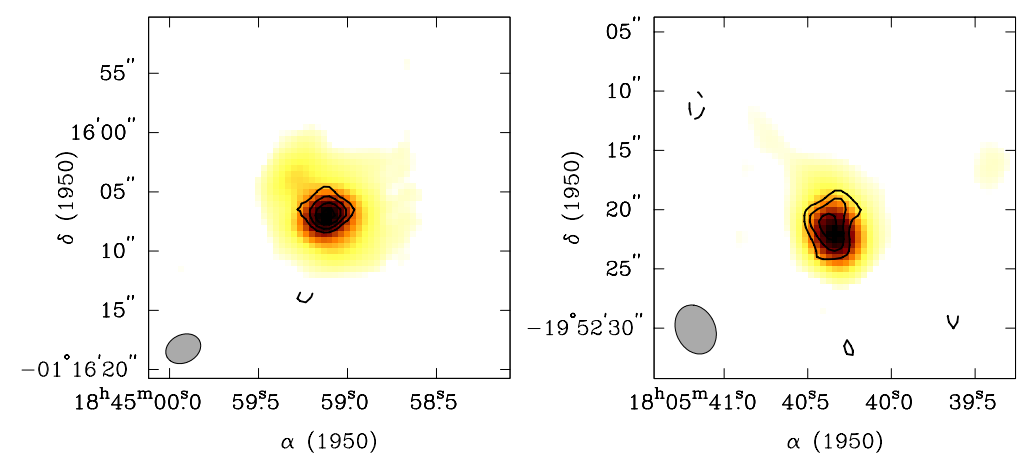

Figure 1. Contours in both panels represent $\mathrm{HCOOH}$ emission while grayscale represent the $3 \mathrm{~mm}$ continuum emission in both regions.

To investigate the distribution of $\mathrm{HCOOH}$ and $\mathrm{CH}_{3} \mathrm{COOH}$, survey observations with the BIMA Array were carried out. $\mathrm{HCOOH}$ was successfully imaged toward the massive star forming cores in Sgr B2 (N), Orion KL, and W51 (Liu, Mehringer, \& Snyder 2001) while $\mathrm{CH}_{3} \mathrm{COOH}$ was mapped in Sgr B2(N) and W51 (Remijan et al. 2002). Further observations carried out with the BIMA Array as well as the OVRO Millimeter Array mapped HCOOH emission in G34.3 + 0.2, G19.61 - 0.23, W75 N (Remijan et al. 2003, 2004) as well as G31.41+0.31 and G10.47+0.03 (Liu et al. 2005a; Fig. 1), respectively. BIMA observations also detected $\mathrm{CH}_{3} \mathrm{COOH}$ toward $\mathrm{G} 34.3+0.2$ (Remijan et al. 2004).

In these observations, both $\mathrm{HCOOH}$ and $\mathrm{CH}_{3} \mathrm{COOH}$ emission was found to be compact and co-spatial with the dust emission from dense cores. The derived $\mathrm{HCOOH}$ abundances of $10^{-10}$ to $10^{-8}$ (Liu, Mehringer, \& Snyder 2001) were at least comparable to, or a few orders of magnitudes higher than those suggested by gas-phase reaction pathways for cold dark clouds such as s L134 N and L183 (Irvine et al. 1990; Turner, Terzieva, \& Herbst 1999). The $\mathrm{CH}_{3} \mathrm{COOH}$ abundances of $10^{-2} \sim 10^{-1}$ relative to that of $\mathrm{HCOOCH}_{3}$, were also found to be much higher than the predictions of pure gas-phase reaction networks. The formation mechanism for $\mathrm{HCOOH}$ and $\mathrm{CH}_{3} \mathrm{COOH}$ detected in these surveys was therefore attributed to grain-surface process. Several grain-surface reaction scenarios (Tielens \& Hagen 1982; Allamandola, \& Sandford 1990; Charnley 1995) in fact suggested $\mathrm{HCOOH}$ readily produced in icy grain mantles. Upon mantle evaporation, $\mathrm{HCOOH}$ will be released into the gas phase.

Recent BIMA observations toward Orion KL at higher angular resolution demonstrated that $\mathrm{HCOOH}$ shows a partial shell morphology (Liu et al. 2002, see Fig. 2). Its unique distribution suggests that $\mathrm{HCOOH}$ is located in a layer that delineates the interaction region between the outflow and the ambient quiescent gas. This is supported by $\mathrm{HCOOH}$ emission being at the southwest rim of numerous $\mathrm{H}_{2} \mathrm{O}$ masers spots as well as a shell of $\mathrm{SO}_{2}$ emission, both considered to be strongly enhanced due to passages of shocks (Kaufman \& Neufeld 1996; Pineau et al. 1993). The HCOOH distribution is also noticeably different from those of other complex O-bearing molecules such as $\mathrm{HCOOCH}_{3}$ and $\left(\mathrm{CH}_{3}\right)_{2} \mathrm{O}$ (Fig. 3), suggesting the former is freshly evaporated from grain surfaces while the latter are likely synthesized from evaporated species via gas-phase routes. It was noted that the observed $\mathrm{HCOOH}$ gas-phase abundances are, however, significantly less than those implied on grain surfaces by IR absorption studies. UV or soft X-ray destruction of $\mathrm{HCOOH}$ in ices or immediately after mantle evaporation has been proposed to account for this (Ehrenfreund et al. 2001; Boechat-Roberty, Pilling, \& Santos 2005). 


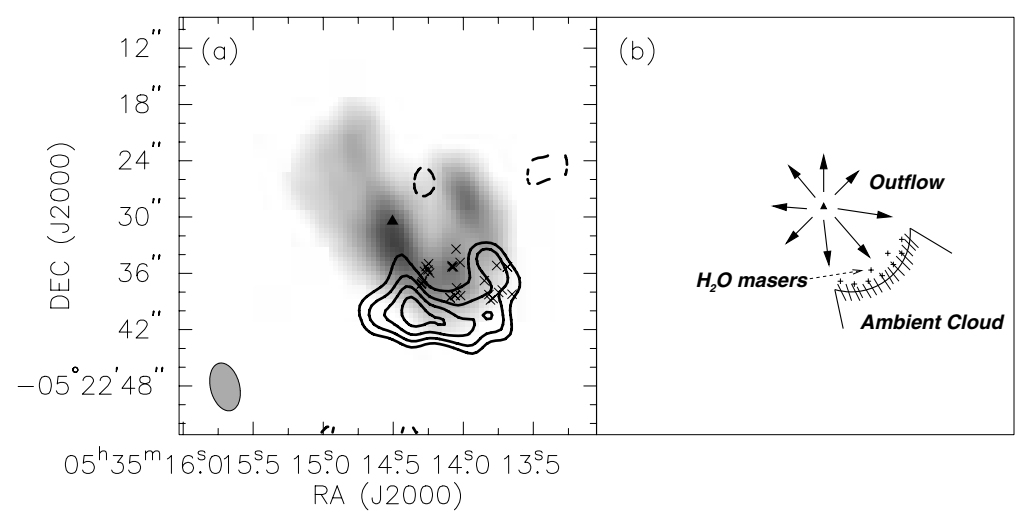

Figure 2. (a) Thick contours represent $\mathrm{HCOOH}$ emission. The $\mathrm{SO}_{2}$ emission is shown in grayscale. The filled triangle denotes the radio continuum source I, while the crosses represent $\mathrm{H}_{2} \mathrm{O}$ maser locations. (b) A diagram illustrating the cloud configuration (Liu 2002).

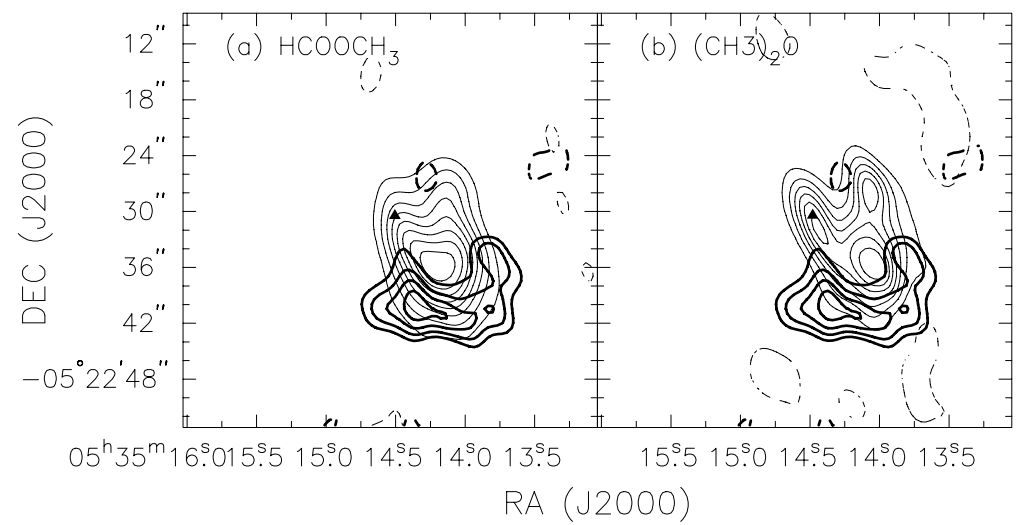

Figure 3. Thick contours in both panels represent $\mathrm{HCOOH}$ emission. (a) Thin contours represent $\mathrm{HCOOCH}_{3}$ emission. (b) Thin contours represent $\left(\mathrm{CH}_{3}\right)_{2} \mathrm{O}$ emission. (Liu 2002).

\section{The Imaging of the $\mathrm{C}_{2} \mathrm{H}_{4} \mathrm{O}$ Isomeric Triplet}

Two important isomeric triplets contain species that are unable to be formed via quiescent gas-phase processes alone: one triplet is the $\mathrm{C}_{2} \mathrm{H}_{4} \mathrm{O}_{2}$ family, including methyl formate $\left(\mathrm{HCOOCH}_{3}\right)$, acetic acid $\left(\mathrm{CH}_{3} \mathrm{COOH}\right)$, and glycolaldehyde $\left(\mathrm{HOCH}_{2} \mathrm{CHO}\right)$; the other is

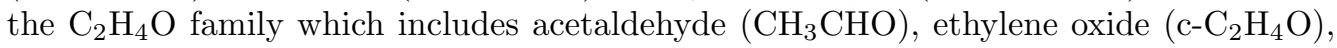
and vinyl alcohol $\left(\mathrm{CH}_{2} \mathrm{CHOH}\right)$. While $\mathrm{HCOOCH}_{3}$ and $\mathrm{CH}_{3} \mathrm{CHO}$ were discovered in the 1970 s, the remaining species $\left(\mathrm{CH}_{3} \mathrm{COOH}, \mathrm{c}-\mathrm{C}_{2} \mathrm{H}_{4} \mathrm{O}, \mathrm{HOCH}{ }_{2} \mathrm{CHO}\right.$, and $\left.\mathrm{CH}_{2} \mathrm{CHOH}\right)$ were identified only fairly recently, all toward Sgr B2 (Mehringer et al. 1997; Dickens et al. 1997; Hollis, Lovas, \& Jewell 2000; Turner \& Apponi 2001). For the $\mathrm{C}_{2} \mathrm{H}_{4} \mathrm{O}_{2}$ family, observations of $\mathrm{HOCH}_{2} \mathrm{CHO}$ with the BIMA array showed, surprisingly, that it existed largely in an extended region toward Sgr B2(N). This led Hollis et al. (2001) to suggest the reactive nature of sugars may account for the $\mathrm{HOCH}_{2} \mathrm{CHO}$ deficiency in the $\mathrm{Sgr}$ B2(N) hot core (see also Hollis, this volume).

To directly probe the spatial distribution of the $\mathrm{C}_{2} \mathrm{H}_{4} \mathrm{O}$ isomeric triplet, observations of these species were carried out toward Sgr B2(N) and Orion KL using the BIMA array (Liu et al. 2005b). Sgr B2(N-LMH) is a uniquely rich hot core region where many complex species, including $\mathrm{c}_{-} \mathrm{C}_{2} \mathrm{H}_{4} \mathrm{O}$ and $\mathrm{CH}_{2} \mathrm{CHOH}$, were first identified. As evident in Figure 4, emission of $\mathrm{c}_{-} \mathrm{C}_{2} \mathrm{H}_{4} \mathrm{O}$ toward $\mathrm{Sgr} \mathrm{B} 2(\mathrm{~N})$ is largely resolved out, indicating that 


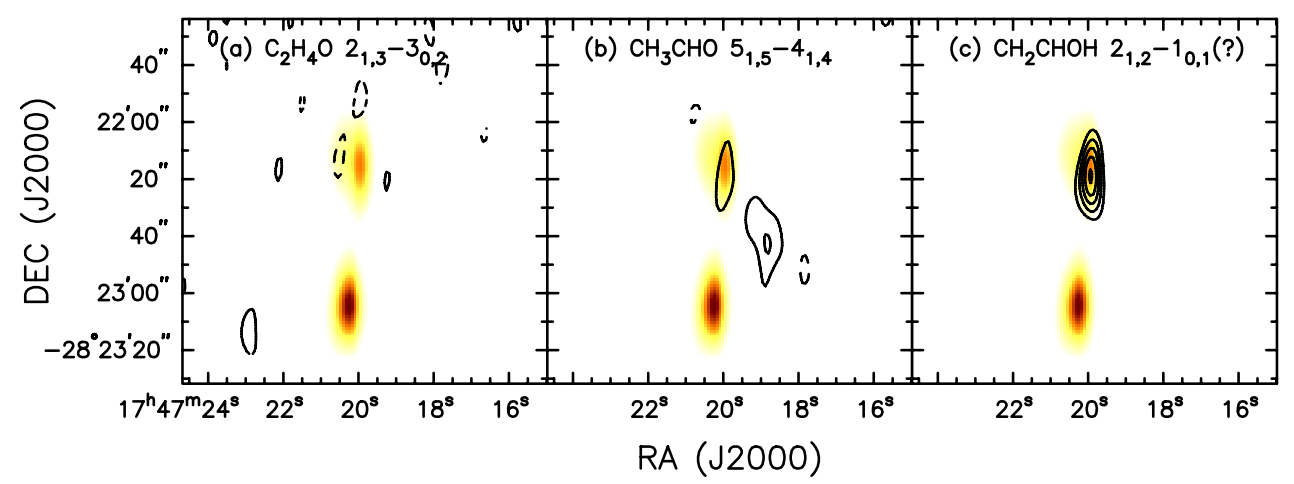

Figure 4. The grayscale in all three panels represents $3 \mathrm{~mm}$ continuum emission toward Sgr B2. (a) Contours represent $c-\mathrm{C}_{2} \mathrm{H}_{4} \mathrm{O}$ emission. (b) Contours represent $\mathrm{CH}_{3} \mathrm{CHO}$ emission. (c) Contours represent possible $\mathrm{CH}_{2} \mathrm{CHOH}$ emission.

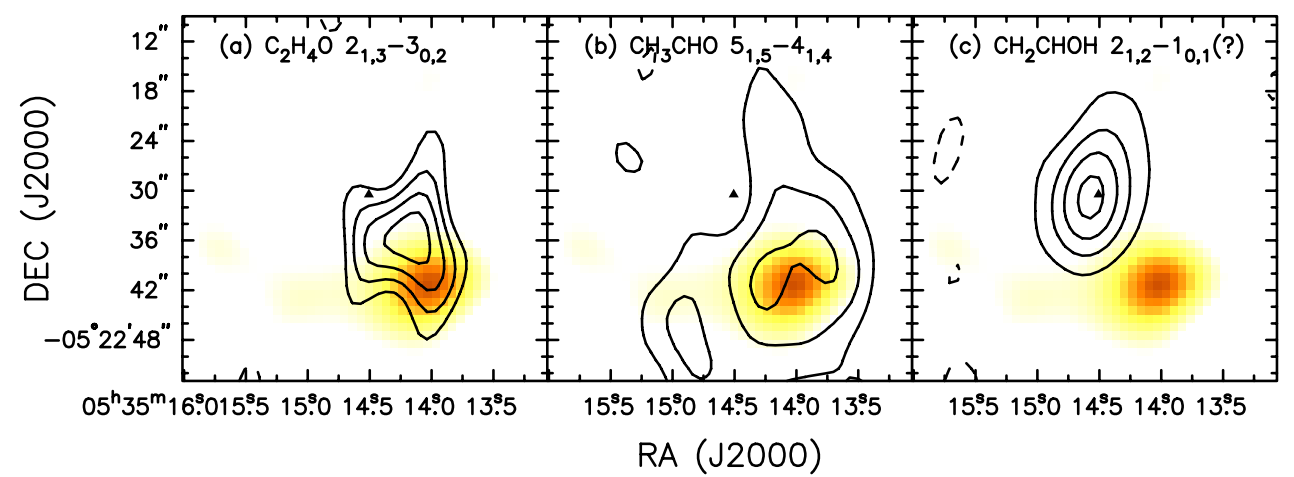

Figure 5. The grayscale in all three panels represents $\mathrm{HCOOH}$ emission toward Orion KL (Liu et al. 2001). (a) Contours represent $\mathrm{c}^{-} \mathrm{C}_{2} \mathrm{H}_{4} \mathrm{O}$ emission. (b) Contours represent $\mathrm{CH}_{3} \mathrm{CHO}$ emission. (c) Contours represent possible $\mathrm{CH}_{2} \mathrm{CHOH}$ emission.

its distribution is rather extended as was suggested by Dickens et al. (1997) based on their observed line-width vs. beam-size relation. $\mathrm{CH}_{3} \mathrm{CHO}$ emission is found to arise from Sgr B2(N), extending toward the south-west. These features are in good agreement with the strongest part of the $\mathrm{c}_{-} \mathrm{C}_{2} \mathrm{H}_{4} \mathrm{O} 1_{1,1}-1_{1,0}$ emission imaged by Chengalur \& Kanekar (2003) with the GMRT. Given that its relative abundance to $\mathrm{H}_{2} \mathrm{CO}$ is considerably smaller than the ratio of $10^{4}$ as expected from pure gas-phase models for the production of these molecules (Lee et al. 1996), Chengalur \& Kanekar suggested that grain chemistry is likely to be important for the enhanced $\mathrm{CH}_{3} \mathrm{CHO}$; its wide-spread distribution is probably related to the presence of numerous shocks in Sgr B2. This is in accordance with the large number of hot expanding shells produced by massive stars in the galactic center region found by Martín-Pintado et al. (1999) and the the quasi-thermal $44 \mathrm{GHz}$ methanol emission also likely arising from shocked gas at the boundaries of expanding ionized shells around the young stars, as suggested by Mehringer \& Menten (1997).

Orion KL is the nearest archetypical hot core source, with the detection of $c_{-} \mathrm{C}_{2} \mathrm{H}_{4} \mathrm{O}$ recently reported by Ikeda et al. (2001). Our interferometric observations with BIMA reveal that the $\mathrm{c}_{-} \mathrm{C}_{2} \mathrm{H}_{4} \mathrm{O}$ emission peaks are offset from the $\mathrm{HCOOH}$ emission feature, but rather coincident with the $\mathrm{HCOOCH}_{3}$ and $\left(\mathrm{CH}_{3}\right)_{2} \mathrm{O}$ emission peaks as shown in Figure 6 (similar to Fig. 3 but with different $\mathrm{HCOOCH}_{3}$ and $\left(\mathrm{CH}_{3}\right)_{2} \mathrm{O}$ transitions and at a lower angular resolution). In contrast, $\mathrm{CH}_{3} \mathrm{CHO}$ emission is mostly located toward the $\mathrm{HCOOH}$ emission peak. The spatial coincidence of the $\mathrm{CH}_{3} \mathrm{CHO}$ emission with the 


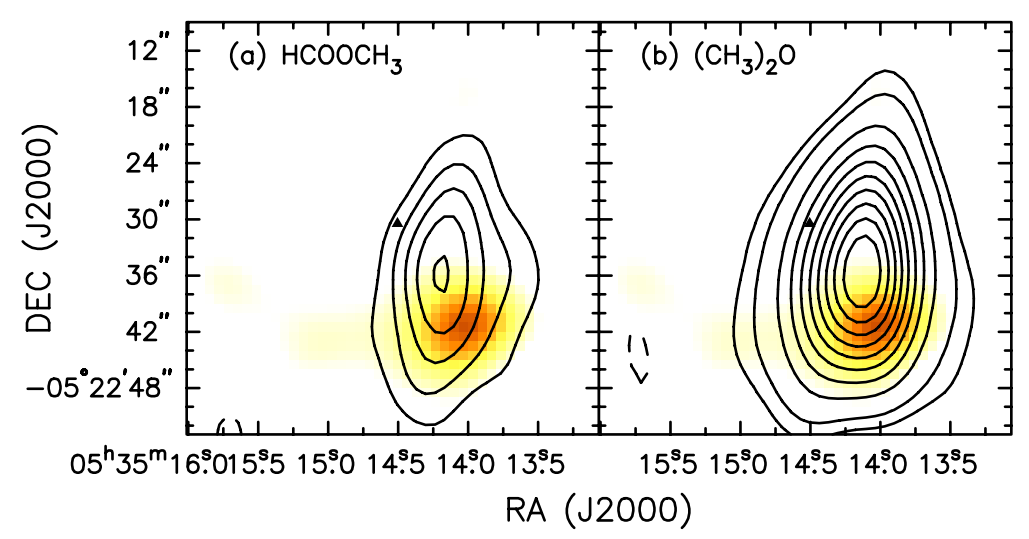

Figure 6. As in Figure 5, the grayscale in both panels depicts the $\mathrm{HCOOH}$ emission toward Orion KL mapped by Liu et al. (2001). (a) Contours represent $\mathrm{HCOOCH}_{3}$ emission. (b) Contours represent $\left(\mathrm{CH}_{3}\right)_{2} \mathrm{O}$ emission.

$\mathrm{HCOOH}$ emission and the $\mathrm{c}-\mathrm{C}_{2} \mathrm{H}_{4} \mathrm{O}$ distribution with the $\mathrm{HCOOCH}_{3}$ as well as $\left(\mathrm{CH}_{3}\right)_{2} \mathrm{O}$ strongly suggests that $\mathrm{CH}_{3} \mathrm{CHO}$ is directly released into the gas phase from mantle evaporation like $\mathrm{HCOOH}$, while $\mathrm{c}^{-} \mathrm{C}_{2} \mathrm{H}_{4} \mathrm{O}$ is more likely a secondary species formed by warm gas phase reactions. Charnley (2004) suggested that pure reduction/hydrogenation reactions of $\mathrm{CO}$ primarily synthesize $\mathrm{CH}_{3} \mathrm{CHO}$ in mantle environments while reducingoxidizing reactions involving acetylene on grain surface are possible pathways for the formation of the $\mathrm{C}_{2} \mathrm{H}_{4} \mathrm{O}$ triplet members. If $\mathrm{CH}_{3} \mathrm{CHO}$ is directly evaporated from gain mantles while $\mathrm{c}_{-} \mathrm{C}_{2} \mathrm{H}_{4} \mathrm{O}$ is a secondary species formed in the gas phase, as suggested by their spatial distribution, this evidence implies that pure reduction/hydrogenation is perhaps the dominate surface process for molecular synthesis on grain surfaces.

\section{The Chemical Differentiation in HMCs}

Past observations have often found chemical differentiation within/among star forming cores. One example is Orion KL. It has long been recognized that considerable differences in the chemical compositions exist between different core components, more specifically, the so called "hot core" and the "compact ridge" (Blake et al. 1987). The "hot core" is pronounced in complex and saturated N-bearing molecules, such as $\mathrm{NH}_{3}, \mathrm{C}_{2} \mathrm{H}_{5} \mathrm{CN}$, and $\mathrm{CH}_{3} \mathrm{CN}$; while the complex O-bearing molecules characterized by $\mathrm{CH}_{3} \mathrm{OH}, \mathrm{HCOOCH}_{3}$, and $\left(\mathrm{CH}_{3}\right)_{2} \mathrm{O}$ are most abundant in the nearby "compact ridge". High angular resolution observations toward the $\mathrm{W} 3(\mathrm{OH}) / \mathrm{W} 3\left(\mathrm{H}_{2} \mathrm{O}\right)$ cores also revealed similar chemical gradients (Wyrowski et al. 1999).

G9.62 + 0.19, located at a distance of $5.7 \mathrm{kpc}$, is a high-mass star forming region associated with a luminous far IR source IRAS $18032-2032\left(3 \times 10^{5} \mathrm{~L}_{\odot}\right)$ that contains a cluster of $\mathrm{H}$ in regions, probably at different evolutionary stages. Multi-wavelength VLA observations identified nine radio continuum sources (denoted A-I) in this region (Garay et al. 1993; Testi et al. 2000). A and B have more extended ( 15-30") morphologies, indicating that they are probably more evolved. C-I are compact sources with angular sizes $<5^{\prime \prime}$ lying along a SE-NW ridge where a chain of $\mathrm{H}_{2} \mathrm{O}$ maser spots were detected by Hofner \& Churchwell (1996). As revealed in $\mathrm{NH}_{3}(4,4),(5,5)$ and $\mathrm{CH}_{3} \mathrm{CN}(J=6-5)$, component $\mathrm{F}$ is a $\mathrm{HMC}$ and hence likely the youngest source in the region (Cesaroni et al. 1994; Hofner et al. 1996). Our observations with the OVRO Millimeter Array and the Submillimeter Array (SMA) show that, while $\mathrm{CH}_{3} \mathrm{CN}$ emission is strongest toward 


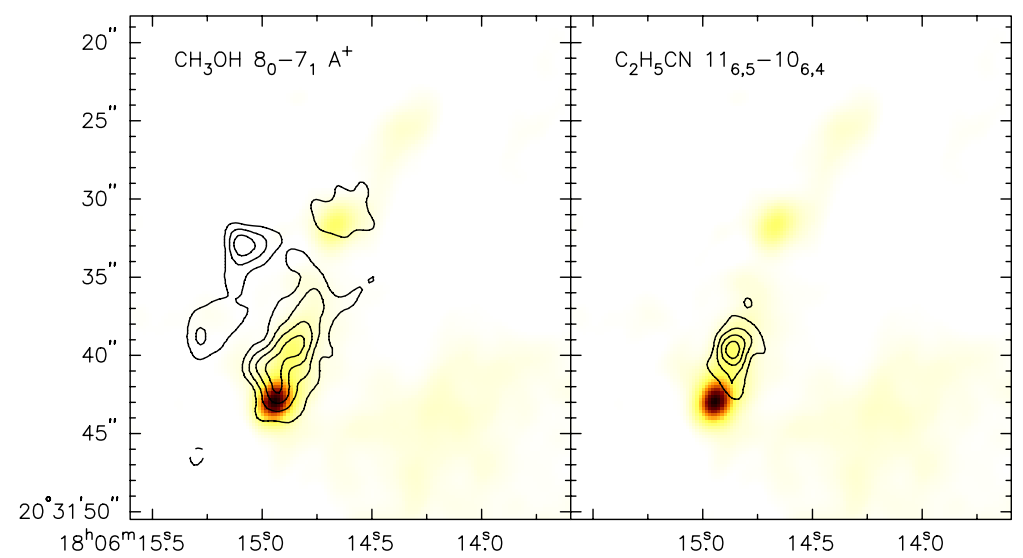

Figure 7. The grayscale in both panel represents $3 \mathrm{~mm}$ continuum emission from G9.62+0.19. (a) Contours represent integrated $\mathrm{CH}_{3} \mathrm{OH} 8_{0}-7_{1} \mathrm{~A}^{+}$line emission. (b) Contours represent integrated $\mathrm{C}_{2} \mathrm{H}_{5} \mathrm{CN} 11_{6,5}-10_{6,4}$ line emission.

the $\mathrm{HMC}-\mathrm{F}$ source, $\mathrm{HCOOCH}_{3}$ and $\left(\mathrm{CH}_{3}\right)_{2} \mathrm{O}$ are brightest in the $\mathrm{H}$ II region $\mathrm{E}$ (see Fig. 7 and 8$)$. The E/F pair in G9.62+ 0.19 thus appears similar to the $\mathrm{W} 3(\mathrm{OH}) / \mathrm{W} 3\left(\mathrm{H}_{2} \mathrm{O}\right)$ system, where a hot core is dominated by N-bearing species and a nearly $\mathrm{H}$ II region is pronounced mainly in emission from O-bearing species. Interestingly, such chemical differentiation is not exclusively found in massive star forming regions, such as those shown by SMA observations toward G9.62+0.19 ( $\mathrm{Su}$ et al. 2005) and Orion KL (Beuther et al. 2005). Recent results from SMA also revealed chemical differentiation in the protobinary low-mass protostar IRAS 16293-2422 (Kuan et al. 2004).

Theoretic models for the origin of such chemical differentiation have been proposed by, for example, Casille, Hasegawa, \& Herbst (1993), Rodgers \& Charnley (2001), and recently by Rodgers \& Charnley (2003). These models suggest that the thermal/evolutionary history of cloud cores may modify grain mantle composition or govern mantle desorption processes differently in various parts of the cloud, and hence result in distinct chemical signatures as a function of location. G9.62 +0.19 , being a region readily displaying chemical differentiation features among various cores/H II regions, warrants future high sensitivity/resolution observations for further verification of these proposed chemical models.

\section{Conclusions}

With the results from studies of $\mathrm{HCOOH}$ and $\mathrm{CH}_{3} \mathrm{COOH}$, as well as those of the $\mathrm{C}_{2} \mathrm{H}_{4} \mathrm{O}$ isomeric triplet, we demonstrate that interferometric observations which spatially resolve molecular emission are powerful tools for distinguishing their formation processes. We also show that G9.62 +0.19 provides an example of chemical differentiation signatures that are possibly related to evolutionary effects.

Future interferometric arrays such as the Atacama Large Millimeter Array (ALMA) with wider bandwidths and more collecting area will be able to carry out similar but much more sensitive, multi-transition observations for diagnosing the chemical structures of molecular cloud cores. We stress that more laboratory measurements and theoretical calculations of molecular rest frequencies are strongly desired for identifying the numerous weak spectral features expected. More sophisticated but user friendly radiative transfer modeling and chemical schemes are also required for better interpreting the vast amount of observational data anticipated. 


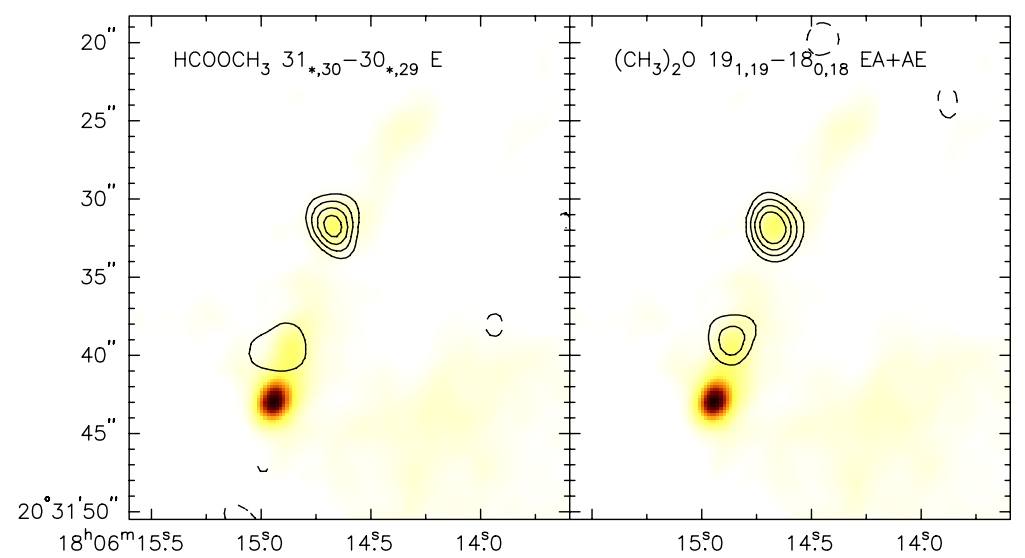

Figure 8. As in Figure 7, the grayscale in both panel represents $3 \mathrm{~mm}$ continuum emission from G9.62+ 0.19. (a) Contours represent integrated $\mathrm{HCOOCH}_{3} 31_{*, 30}-30_{*, 29}$ line emission. (b) Contours represent integrated $\left(\mathrm{CH}_{3}\right)_{2} \mathrm{O} 19_{1,19}-18_{0,18} \mathrm{EA}+\mathrm{AE}$ line emission.

\section{Acknowledgements}

S.-Y. Liu is grateful to the Scientific Organizing Committee for the invitation of make this presentation. Many thanks also go to L.E. Snyder, D. Mehringer, A. Remijan, G.A. Blake, Y.-J. Kuan, and Y.-N. Su for collaboration on the studies presented here.

\section{References}

Allamandola, L.J. \& Sandford, S.A. 1990, NASA Conf. Pub. 3061, 113

Blake, G.A., Sutton, E.C., Masson, C.R., \& Phillips, T.G. 1987, Ap. J. 315, 621

Beuther, H., et al. 2005, Ap. J., in press

Boechat-Roberty, H.M., Pilling, S., \& Santos, A.C.F. 2005, A\&̋A 438, 915

Caselli, P., Hasegawa, T.I., \& Herbst, E. 1993, Ap. J. 408, 548

Cesaroni, R., Churchwell, E., Hofner, P., Walmsley, C.M., \& Kurtz, S. 1994, A\&\&A 288, 903

Charnley, S.B. 1995, Ap\&SS, 224, 251

Charnley, S.B. 2004, ASR, 33, 23

Chengalur, J.N. \& Kanekar, N. 2003, A\&A 403, L43

Crovisier, J. \& Bockelée-Morvan, D. 1999, Space Scien. Rev., 90, 19

Dickens, J.E., Irvine, W.M., Ohishi, M., Ikeda, M., Ishikawa, S., Nummelin, A., \& Hjalmarson, A. 1997, Ap. J. 489, 753

Ehrenfreund, P., D'Hendecourt, L., Charnley, S., \& Ruiterkamp, R. 2001, JGR, 106, 33291

Garay, G., Rodriguez, L.F., Moran, J.M., \& Churchwell, E. 1993, Ap. J. 418, 368

Hollis, J.M., Lovas, F.J., \& Jewell, P.R. 2000, Ap. J. 540, L107

Hollis, J.M., Vogel, S.N., Snyder, L.E., Jewell, P.R., \& Lovas, F.J. 2001, Ap. J. 554, L81

Hofner, P., Kurtz, S., Churchwell, E., Walmsley, C.M., \& Cesaroni, R. 1996, Ap. J. 460, 359

Ikeda, M., Ohishi, M., Nummelin, A., Dickens, J.E., Bergman, p., Hjalmarson, Å., \& Irvine, W.M. 2001, Ap. J. 560, 792

Irvine, W.M., Friberg, P., Kaifu, N., Matthews, H.E., Minh, Y.C., Ohishi,M., \& Ishikawa, S. 1990, A\&SA 229, L9

Kaufman, M.J. \& Neuleld, D.A. 1996, Ap. J. 456, L611

Kurtz, S., Cesaroni, R., Churchwell, E., Hofner, P., \& Walmsley, C.M. 2000, in Ptostars and Planets IV, ed. V. Manning, A.P. Boss, \& S. Russell (Tucson: Univ. Arizona Press), 299

Kuan, Y.-J., et al. 2004, Ap. J. 616, L27

Lee, H.-H., Bettens, R.P.A., \& Herbest, E. 1996, A\&AS, 119, 111

Liu, S.-Y., Mehringer, D.M., \& Snyder, L.E. 2001, Ap. J. 552, 654

Liu, S.-Y., Girart, J.M., Remijan, A., \& Snyder, L.E. 2002, Ap. J. 576, 255 
Liu, S.-Y., et al. 2005a, in preparation

Liu, S.-Y., et al. 2005b, in preparation

Martín-Pintado, J., Gaume, R.A., Rodríguez-Fernández, N., de Vicente, P., \& Wilson, T.L. 1999, Ap. J. 519, 667

Mehringer, D.M. \& Menten, K.M. 1997, Ap. J. 474, 346

Mehringer, D.M. \& Snyder, L.E. 1996, Ap. J. 471, 897

Mehringer, D.M., Snyder, L.E., Miao, Y., \& Lovas, F.J. 1997, Ap. J. 480, L71

Miao, Y., Mehringer, D.M., Kuan, Y.-J., \& Snyder, L.E. 1995, Ap. J. 445, L59

Millar, T.J., Olofsson, H., Hjalmarson, Å., \& Brown, R.D. 1988, A $ళ$ A 205, L5

Pineau des Forêts, G., Roueff, E., Schilke, P., \& Flower, D.R. 1993, MNRAS, 262, 915

Remijan, A.J., Liu, S.-Y., Snyder, L.E., Mehringer, D.M., \& Kuan, Y.J. 2002, Ap. J. 576, 264

Remijan, A., Shiao, Y.-S., Fridel, D.N., Meier, D.S., \& Snyder, L.E. 2004, Ap. J. 617, 384

Remijan, A., Snyder, L.E., Friedel, D.N., Liu, S.-Y., \& Shah, R.Y. 2003, Ap. J. 590, 314

Rodgers, S.D. \& Charnley, S.B. 2001, Ap. J. 546, 324

Rodgers, S.D. \& Charnley, S.B. 2003, Ap. J. 585, 355

Schilke, P. Groesbeck, T.D., Blake, G.A., \& Phillips, T.G. 1997, Ap. J.S, 108, 301

Schutte, W.A., et al. 1996, A\&A 315, L333

Schutte, W.A., et al. 1999, A\&A 343, 966

$\mathrm{Su}, \mathrm{Y} .-\mathrm{N}$. , et al. 2005, in preparation

Testi, L., Hofner, P., Kurtz, S., \& Rupen, M. 2000, A\& A 359, L5

Tielens, A.G.G. \& Hagen, W. 1982, A\& A 114, 245

Turner, B.E. 1991, Ap. J. Suppl. 76, 617

Turner, B.E. \& Apponi, A.J. 2001, Ap. J. 561, L207

Turner, B.E., Terzieva, R., \& Herbst, E. 1999, Ap. J. 518, 699

Winnewisser \& Churchwell 1975, Ap. J. 200, L33

Wyrowski, F. Schilke, P., Walmsley, C.M., \& Menten, K.M. 1999, Ap. J. 514, L43

Ziurys, L.M. \& McGonagle, D. 1993, Ap. J. Suppl. 89, 155

Zuckermann, B., Ball, J.A., \& Gottlieb, A.C. 1971, Ap. J. 163, L41

\section{Discussion}

HERBST: How does interferometry help identify new complex molecules?

LIU: Interferometers provide both spectral as well as spatial information on line features. They therefore assist in new line identification only if all potential spectral features match both in frequency (velocity) and in their spatial distribution (existing in the same volume) with the correct corresponding velocity. Interferometers additionally filter out spectral line originated from extended structures which may otherwise blank or confuse weak target lines in single-dish telescope surveys.

van Dishoeck: How much do the column densities and - in particular- the column density/abundance ratios of complex molecules change when derived from interferometer data compared with single-dish observations (in your experience)?

LiU: Single-dish observations provide only abundances and (abundance) ratios averaged over the beam. Depending on the source structure, the molecular column densities can be modified (typically increased) by large factors when observed with interferometers. The relative abundance ratios between different species can also change drastically, on occassion by orders of magnitude. This is particularly true if there is a spatial differentiation between species, such as is the case for $\mathrm{c}-\mathrm{C}_{2} \mathrm{H}_{4} \mathrm{O} / \mathrm{CH}_{3} \mathrm{CHO}$ toward Orion, as we demonstrate here. 


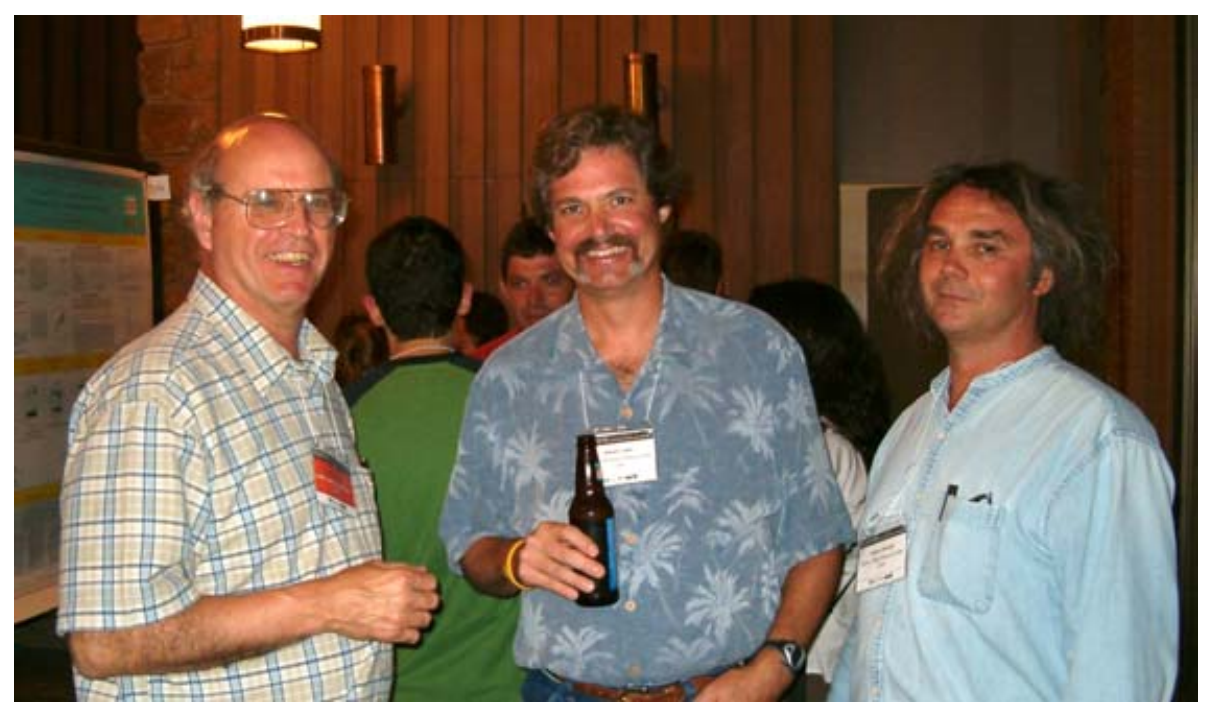

Photo: E. Herbst 